\title{
New Editorial Board for Clinical and Translational Oncology
}

\author{
Juan Carlos Lacal
}

$\mathrm{T}^{\mathrm{s}}$ he recent achievements of our journal, with its acceptance into the Science Citation Index Expanded (SciSearch) and JCR/Science Edition, are indeed an important step forward that encompasses the definition of new objectives, wishes and aims. Once the principal goal of this phase has been successfully achieved, it will be time to devise new strategies and to gather the right tools to implement them. Our major focus for the next few years will be to further improve the quality of the journal. This new scenario has prompted the Executive Committee of the Federation of Spanish Cancer Societies (FESEO) to introduce some adjustments to the Editorial Board that will take place starting from the present issue. Guided by a renovated interest in providing the necessary support and strength to guarantee the new envisioned goals, a few but very significant changes have been introduced.

First, according to the personal desire of the Editor-in-Chief, Dr. Miguel Martin, to be released from his responsibilities in the journal, Dr. Andrés Cervantes has been promoted to this post. It is indeed with our great appreciation and recognition of his effort and contribution for more than seven years that we thank Dr. Martin for his performance and input to the enormous improvements that the journal has experienced during his service as Editor-in-Chief. This important position is now taken over by another prestigious medical oncologist, Dr. Cervantes, who will continue Dr, Martin's contribution with a complementary and personal commitment. Although the achievements and responsibilities of the position of Editor-in-Chief have been set at a very high level, we are convinced that with the appointment of Dr. Cervantes the journal will maintain its successful path, will continue improving and will reach its new envisioned aims.

Another very significant change is the incorporation of Dr. Antonio Llombart, a very well known young, but experienced, medical oncologist who joins the Editorial Board with the responsibilities of Deputy Editor, a newly ex profeso created position. His main task will be to provide the mechanisms and resources to build a stronger bridge between the clinical oncology

Executive Editor

J.C. Lacal (凶)

Centro Nacional de Biotecnología

C/ Darwin, 3

Campus de Cantoblanco

ES-28049 Madrid, Spain

e-mail: jclacal@cnb.csic.es world and the basic oncology world. This is a renewed effort to reinforce even further the determination of Clinical and Translational Oncology to promote Translational Oncology as one of its major commitments and services to the oncology community.

With these necessary adjustments in the Editorial Board, we hope that the journal will gain a renovated impulse towards new and very ambitious goals. A brief description of these is given in the following lines.

Clinical and Translational Oncology will maintain its efforts to bring to our readers, in a clear, easy to read and educational format, the most recent advances that will help understanding of the molecular and cellular biology of tumours and the efforts to identify new drug targets as well as the development of new therapeutic strategies against specific tumours actually in progress both at the laboratory and the clinical levels. Finally, the technological improvements that provide the necessary tools to progress both in research and treatment of cancer will also be covered in an effort to facilitate the continuous update of researchers and clinicians. This effort will allow Clinical and Translational Oncology to gain clear recognition and leadership in the transition from Clinical Oncology to Translational Oncology at the international level.

In addition, a special effort will be dedicated to complement these Educational Series in Translational Oncology with multidisciplinary clinical revisions dedicated to most kinds of tumours. This new Series will help to update clinical oncologists on recent advances, clinical consensus, specialist conferences and new trends in the management of cancer patients.

Finally, but no less important, we will continue to pay special attention to further improvement of the quality and relevance of the original research articles that will be accepted for publication. This represents our special commitment to increase the international impact and visibility of the journal. To improve further in this extremely sensitive and important issue, our "peer review" system will be reinforced without risking our turnaround times from reception to final acceptance and publication. Publication of only high quality research both on the clinical and experimental sides will be the major interest. Special attention will be devoted to attracting cutting edge Translational Oncology as the major body of accepted Research Articles. In this regard, only exceptional, high quality Case Reports will be accepted for publication, especially those that are highly innovative and dedicated to closing the gap between clinical and basic research.

All these adjustments are necessary to further the progress of Clinical and Translational Oncology, at least in the immediate future. We face a very exciting and challenging time that can put the journal in an important position among all the international oncology journals. 